\title{
Reduced expression of Rho GDP dissociation inhibitor 2 mRNA is associated with lymph node metastasis in gastric carcinoma
}

\author{
ATSUO SHIDA, SHUICHI FUJIOKA, NAOTO TAKAHASHI, HIROAKI AOKI, TOMOYOSHI OKAMOTO, \\ NORIO MITSUMORI, NOBUO OMURA and KATSUHIKO YANAGA
}

Department of Surgery, Jikei University School of Medicine, Minato-ku, Tokyo 105-8461, Japan

Received December 19, 2012; Accepted May 20, 2013

DOI: 10.3892/ol.2013.1379

\begin{abstract}
Small GTPase proteins, including RhoA, RhoB, RhoC, Rac1 and cdc42, are molecules that have significant roles in linking cell shape and cell cycle progression in cytoskeletal arrangements and mitogenic signaling. Rho GDP dissociation inhibitor 2 (RhoGDI2) has recently been identified as a metastasis suppressor gene in models of bladder cancer. RhoGDI2 has also been identified as a potential regulator of tumorigenesis and cancer progression. The present study aimed to clarify the significance of RhoGDI 2 gene expression in gastric carcinoma and to evaluate the outcome of affected patients. A total of 46 pairs of normal mucosa and cancer specimens were obtained from patients who had undergone a gastrectomy for primary gastric carcinoma and were subjected to semi-quantitative reverse transcription polymerase chain reaction (RT-PCR) for RhoGDI2. The expression of RhoGDI2 mRNA was significantly higher in early-stage gastric cancer specimens compared with the normal gastric epithelium samples. By contrast, the depth of the tumor was negatively correlated with RhoGDI 2 mRNA expression. In addition, a reduced expression of RhoGDI 2 mRNA was associated with venous system invasion and lymph node metastasis. RhoGDI 2 mRNA was more frequently expressed in differentiated adenocarcinoma compared with poorly-differentiated adenocarcinoma. Although the statistical significance was not established, RhoGDI2-positive patients tended to have a superior oncological outcome compared with RhoGDI2-negative patients. The reduced expression of RhoGDI 2 mRNA in gastric carcinoma is associated with venous system invasion and lymph node metastasis.
\end{abstract}

\section{Introduction}

The Rho GTPases, which are from a distinct branch of the Ras-like low molecular weight GTP-binding protein super-

Correspondence to: Dr Atsuo Shida, Department of Surgery, Jikei University School of Medicine, 3-25-8 Nishi-shinbashi, Minato-ku, Tokyo 105-8461, Japan

E-mail: atsuoshida@ybb.ne.jp

Key words: Rho GDP dissociation inhibitor 2, gastric carcinoma family, are involved in actin cytoskeleton organization (1) and have been associated with invasion and metastasis (2). RhoGTPases alternate between an inactive GDP-bound state and an active GTP-bound state. The regulators of this GDP/GTP cycle include GDP dissociation inhibitors (GDIs), which bind to the inactive form, thereby blocking further activation. RhoGDI1 was first identified on the basis of its ability to inhibit GDP dissociation from RhoA (3), CDC42Hs (4) and Rac1 (5). RhoGDI2, also known as D4-GDI or Ly-GDI, shares a $67 \%$ amino acid analogy with RhoGDI1 (6-8). However, in contrast with the ubiquitous property of RhoGDI1, the protein is believed to be expressed exclusively on cells of hematopoietic lineage (6,7). However, Seraj et al (9) and Gildea et al (10) suggested that the RhoGDI2 gene is also expressed in non-hematopoietic neoplasms. Furthermore, Gildea et al (11) have shown using an animal model of human bladder cancer metastasis and DNA microarray technology that RhoGDI2 is a putative metastasis suppressor gene in human cancer. By analyzing patient immunohistochemistry (IHC), Theodorescu et al reported that RhoGDI2 is an independent predictor of prognosis for patients with bladder cancer (12). Also, Hu et al (13) demonstrated that the reduced expression of RhoGDI 2 in breast cancer was associated with lymph node metastasis. To date, there have been no studies on the impact of RhoGDI2 mRNA expression for the survival of patients with gastric carcinoma. Hence, the present study investigated the expression of RhoGDI 2 in human gastric cancer specimens and evaluated the significance of RhoGDI2 expression on the outcome and clinicopathological parameters of the patients.

\section{Patients and methods}

Patients and tumor samples. A total of 46 patients who had undergone a gastrectomy with lymph node dissection for primary gastric carcinoma at Jikei University Daisan Hospital (Tokyo, Japan) and Shiomidai Prefectural Hospital (Kanagawa, Japan) between March 2006 and January 2010 were studied. Pre-operative informed consent was obtained from each patient in accordance with institutional guidance. Tumor specimens and normal mucosal tissues were stored at $4^{\circ} \mathrm{C}$ in an RNA preserving reagent (RNA-later, Ambion, Austin, TX, USA). The extraction of total RNA from the samples was performed within three months of tissue extraction. The total RNA was immediately transcribed to first-strand cDNA (1st Strand cDNA 
Synthesis kit; Roche, Basel, Switzerland), which was stored at $-80^{\circ} \mathrm{C}$ until the reverse transcription-polymerase chain reaction (RT-PCR). RT-PCR for RhoGDI2 and glyceraldehyde 3-phosphate dehydrogenase (GAPDH) was performed between July 2011 and August 2011. The RhoGDI2 and GAPDH mRNA levels were quantified using NIH Image 1.63 computer software (Wayne Rasband, NIH, Bethesda, MD, USA), and the intensity of RhoGDI2 for GAPDH was calculated. Pathological and clinical records were reviewed and the disease stage was determined according to the classification by the Japanese Research Society for gastric cancer (14). The histological grading and depth of invasion of the tumors in all the cases were available and are summarized in Table I. The presence of liver metastasis and peritoneal dissemination was determined pre-operatively using radiographic examinations or intra-operatively. Based on the histological grade, the tumor specimens were classified into two groups consisting of the differentiated group (wellto moderately-differentiated adenocarcinoma and papillary adenocarcinoma) and the undifferentiated group (poorly-differentiated adenocarcinoma and signet-ring cell or mucinous carcinoma). This study was approved by the ethics committee of Jikei University School of Medicine, Tokyo, Japan.

Semi-quantitative RT-PCR. Total RNA was extracted from the resected gastric carcinoma and normal tissue specimens using the RNeasy Mini kit (Qiagen, Valencia, CA, USA), according to the manufacturer's instructions. The first-strand cDNA was synthesized from total RNA by reverse transcriptase (1st Strand cDNA Synthesis kit; Roche, Basel, Switzerland). The PCR analysis was performed using the following pairs of primers: RhoGDI2 forward, 5'-agtacgacgtgatcgtgctg-3' and reverse, 5'-gcgagcaatttctcettcag-3'; and GAPDH forward, 5'-atcatccetgcctctactgg-3' and reverse, 5'-ccctccgacgcetgcttcac-3'. Following this, $1 \mu \mathrm{g} / 1$ of the cDNA reaction was subjected to $35 \mathrm{PCR}$ cycles (denaturing at $94^{\circ} \mathrm{C}$ for $1 \mathrm{~min}$, annealing at $58^{\circ} \mathrm{C}$ for $50 \mathrm{sec}$ and polymerization at $72^{\circ} \mathrm{C}$ for $1 \mathrm{~min}$ ), in the presence of $0.25 \mathrm{U}$ Taq DNA Polymerase (Roche, Indianapolis, IN, USA), 1X PCR reaction buffer (Roche), $0.25 \mathrm{mM}$ dNTPs (Promega, Madison, WI, USA) and $0.5 \mu \mathrm{M}$ specific primers for RhoGDI2 and GAPDH in a final reaction volume of $50 \mu \mathrm{l}$.

$I H C$. Using paraffin-embedded specimens from eight patients with gastric cancer, the RhoGDI2 protein was detected using the anti-RhoGDI2 rabbit polyclonal antibody (Abcam, Cambridge, UK). Briefly, subsequent to being microwaved in citrate buffer solution ( $\mathrm{pH}$ 6.0), the deparaffinized sections were incubated with $1 \%$ methanol-hydrogen peroxide for $30 \mathrm{~min}$. The slides were then incubated with the rabbit polyclonal antibody against RhoGDI2 (undiluted solution) for $60 \mathrm{~min}$. This was followed by incubation with anti-rabbit secondary antibody (Envision ${ }^{\mathrm{TM}} /$ Rabbit/HRP; Dako, Carpinteria, CA, USA) for $30 \mathrm{~min}$. The staining was visualized using the diaminobenzidine (DAB) method (Dako) for 5 min. Counter-staining was performed lightly with hematoxylin. All incubations were performed at room temperature in a humidified chamber. Control rabbit immunoglobulin $\mathrm{G}$ was used for each staining (Daiichi Fine Chemical, Takaoka, Toyama, Japan).

Statistics. The significance of the data was determined using the chi-square test or Student's t-test. The multivariate analysis
Table I. Clinical and pathological characteristics of the patients and tumors.

\begin{tabular}{|c|c|}
\hline Characteristic & Value \\
\hline Median age, years & 73 \\
\hline Gender, n (male:female) & $31: 15$ \\
\hline Median size, $\mathrm{mm}$ & 40 \\
\hline \multicolumn{2}{|l|}{ Histological type, n (\%) } \\
\hline Differentiated & $27(59)$ \\
\hline Undifferentiated & $19(41)$ \\
\hline \multicolumn{2}{|l|}{ Location of tumor, n (\%) } \\
\hline $\mathrm{U}$ & $10(22)$ \\
\hline Mid & $25(54)$ \\
\hline $\mathrm{L}$ & $11(24)$ \\
\hline \multicolumn{2}{|l|}{ Gross form, $\mathrm{n}(\%)$} \\
\hline $0-\mathrm{I}$ & $1(2)$ \\
\hline 0-IIa & $7(15)$ \\
\hline $0-\mathrm{IIb}$ & $1(2)$ \\
\hline 0-IIc & $8(17)$ \\
\hline 0 -III & $0(0)$ \\
\hline Type 1 & $2(4)$ \\
\hline Type 2 & $12(26)$ \\
\hline Type 3 & $14(30)$ \\
\hline Type 4 & $1(2)$ \\
\hline \multicolumn{2}{|l|}{ Depth of invasion, n (\%) } \\
\hline pT1 (M, SM) & $15(32)$ \\
\hline pT2 (MP, SS) & $20(43)$ \\
\hline pT3 (SE) & $11(24)$ \\
\hline pT4 (SI) & $0(0)$ \\
\hline Venous invasion ${ }^{+}, \mathrm{n}(\%)$ & $20(43)$ \\
\hline Lymphatic invasion $^{+}, \mathrm{n}(\%)$ & $36(78)$ \\
\hline Lymph node metastasis ${ }^{+}, \mathrm{n}(\%)$ & $25(54)$ \\
\hline Peritoneal dissemination ${ }^{+}, \mathrm{n}(\%)$ & $3(7)$ \\
\hline Synchronous liver metastasis ${ }^{+}, \mathrm{n}(\%)$ & $1(2)$ \\
\hline Synchronous lung metastasis ${ }^{+}, \mathrm{n}(\%)$ & $0(0)$ \\
\hline
\end{tabular}

U, upper; Mid, middle; L, lower; pT, pathological tumor depth; M, mucosa; SM, submucosa; MP, muscularis propia; SS, subserosa; SE, serosa exposed; SI, other organ invasion.

for patient prognosis was determined using the Cox proportional hazards model. The survival curves of the patients were compared using the Kaplan-Meier method and analyzed by the $\log$-rank test. $\mathrm{P}<0.05$ was considered to indicate a statistically significant difference.

\section{Results}

Expression of RhoGDI2 mRNA in human gastric carcinoma tissues

Semi-quantitative RT-PCR. A total of 46 pairs of tissue samples obtained from the tumors of patients with gastric carcinoma and the adjacent non-cancerous mucosa were 
Table II. Correlation between clinicopathological observations and RhoGDI2 expression.

\begin{tabular}{|c|c|c|c|}
\hline \multirow[b]{2}{*}{ Findings } & \multicolumn{2}{|c|}{ RhoGDI2 expression } & \multirow[b]{2}{*}{ P-value } \\
\hline & Positive, $\mathrm{n}$ & Negative, $\mathrm{n}$ & \\
\hline \multicolumn{4}{|l|}{ Histological type } \\
\hline Differentiated & 11 & 16 & \\
\hline Undifferentiated & 3 & 16 & 0.1374 \\
\hline \multicolumn{4}{|l|}{ Location of tumor } \\
\hline $\mathrm{U}$ & 3 & 7 & \\
\hline M & 10 & 15 & \\
\hline $\mathrm{L}$ & 1 & 10 & 0.1555 \\
\hline \multicolumn{4}{|l|}{ Gross form } \\
\hline $0-\mathrm{I}$ & 1 & 0 & \\
\hline 0-IIa & 3 & 4 & \\
\hline 0 -IIb & 1 & 0 & \\
\hline 0 -IIc & 3 & 5 & \\
\hline 0-III & 0 & 0 & \\
\hline Type 1 & 2 & 0 & \\
\hline Type 2 & 2 & 10 & \\
\hline Type 3 & 2 & 12 & \\
\hline Type 4 & 0 & 1 & 0.1214 \\
\hline \multicolumn{4}{|l|}{ Venous invasion } \\
\hline Positive & 3 & 19 & \\
\hline Negative & 11 & 13 & 0.0404 \\
\hline \multicolumn{4}{|l|}{ Lymphatic invasion } \\
\hline Positive & 8 & 22 & \\
\hline Negative & 6 & 10 & 0.4469 \\
\hline \multicolumn{4}{|c|}{ Lymph node metastasis } \\
\hline Positive & 3 & 23 & \\
\hline Negative & 11 & 9 & 0.0043 \\
\hline
\end{tabular}

U, upper; M, middle; L, lower; RhoGDPI2, Rho GDP dissociation inhibitor 2 .

examined for RhoGDI2 gene expression using RT-PCR (Fig. 1).

The 95\% CI [the average ratio + two standard deviations (SD)] was used to select a cut-off line. The mean intensity of RhoGDI2 for GAPDH in 46 normal gastric tissues was 0.01 and the SD was 0.057 . Hence, the cut-off value of RhoGDI2 was defined as 0.124 . According to this cut-off line, RhoGDI2-positive expression was observed in 14 (30.4\%) human gastric carcinoma samples and in one $(2.2 \%)$ normal gastric mucosa sample. The expression of RhoGDI2 mRNA was significantly higher in the early-stage gastric cancer samples compared with the normal gastric mucosa or advanced gastric cancer tissues (Fig. 2; Student's t-test, $\mathrm{P}<0.01$ ). A univariate analysis was carried out to determine the correlation between the conventional pathological prognostic markers and RhoGDI2 mRNA expression. The results show that a reduced expression of RhoGDI2 is associated with venous system invasion and lymph node metastasis (Table II). The tumor expression of RhoGDI2 was further evaluated


C

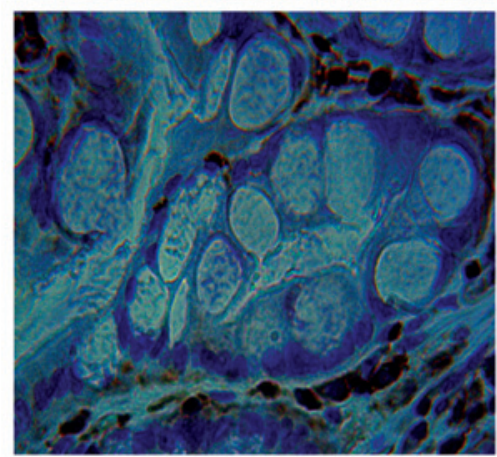

Figure 1. (A) RT-PCR analysis of RhoGDI2 and GAPDH mRNA in two human gastric carcinoma patients. In patient 25, RhoGDI2 mRNA was not expressed in the $\mathrm{T}$ or $\mathrm{N}$ mucosa. However, in patient 26 , RhoGDI2 mRNA was expressed in the $\mathrm{T}$ but not the $\mathrm{N}$ mucosa. (B) Representative IHC staining with RhoGDI2 polyclonal antibody in gastric cancer, showing strong staining for RhoGDI2 in the cytoplasm of the tumor tissue $(\times 400)$. (C) In the N gastric mucosa, RhoGDI2 was not observed in the epithelial cells $(\times 400)$. The samples shown in (B) and (C) are derived from patient 26. RT-PCR, reverse transcription-polymerase chain reaction; RhoGDI2, Rho GDP dissociation inhibitor 2; GAPDH, glyceraldehyde-phosphate dehydrogenase; T, tumor; $\mathrm{N}$, normal; IHC, immunohistochemistry.

as a prognostic variable in patients with gastric carcinoma. Table III and IV show the multivariate analysis, which identified the fact that RhoGDI2 expression was not an independent prognostic factor for relapse-free survival (RFS) or overall survival (OS). However, the data show that RhoGDI2-positive patients had a good prognosis compared with those who were RhoGDI2-negative (Figs. 3 and 4).

$I H C$. The expression of the RhoGDI2 protein was assessed in eight cases of surgically removed gastric tissues using a polyclonal antibody that was specific to RhoGDI2. In several cases, strong staining for RhoGDI2 was observed in the cytoplasm of the tumor tissues, even though negative or very weak RhoGDI2 was observed in the normal gastric tissues.

These IHC examinations corresponded with the results from the RT-PCR (Fig. 1). 
Table III. Risk factors affecting RFS as determined by the Cox proportional hazards model in 40 patients with gastric cancer.

\begin{tabular}{lccr}
\hline & \multicolumn{3}{c}{ Multivariate analysis for RFS } \\
\cline { 2 - 4 } Variable & Hazard ratio & 95\% CI & P-value \\
\hline pT (T1 vs. T2/T3/T4) & 0.359 & $0.153-0.843$ & 0.019 \\
Venous system invasion (yes vs. no) & 0.723 & $0.327-1.597$ & 0.422 \\
Lymphatic system invasion (yes vs. no) & 1.174 & $0.431-3.198$ & 0.754 \\
Lymph node metastasis (yes vs. no) & 1.289 & $0.522-3.179$ & 0.582 \\
RhoGDI2 (positive vs. negative) & 0.641 & $0.264-1.556$ & 0.326 \\
\hline
\end{tabular}

RFS, relapse-free survival; pT, pathological tumor depth; RhoGDI2, Rho GDP dissociation inhibitor 2.

Table IV. Risk factors affecting OS rate determined by Cox proportional Hazards model in 46 patients with gastric cancer.

\begin{tabular}{lccr}
\hline & \multicolumn{3}{c}{ Multivariate analysis for OS } \\
\cline { 2 - 4 } Variable & Hazard ratio & $95 \%$ CI & P-value \\
\hline pT (T1 vs. T2/T3/T4) & 0.399 & $0.166-0.960$ & 0.040 \\
Venous system invasion (yes vs. no) & 0.826 & $0.374-1.824$ & 0.637 \\
Lymphatic system invasion (yes vs. no) & 1.229 & $0.441-3.426$ & 0.692 \\
Lymph node metastasis (yes vs. no) & 1.188 & $0.453-3.117$ & 0.726 \\
RhoGDI2 (positive vs. negative) & 0.516 & $0.211-1.263$ & 0.147 \\
\hline
\end{tabular}

OS, overall survival; pT, pathological tumor depth; RhoGDI2, Rho GDP dissociation inhibitor 2.

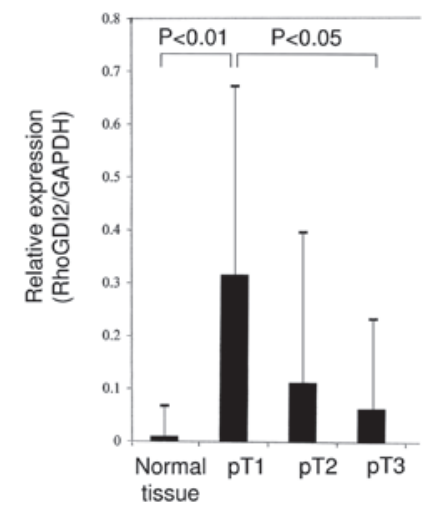

Figure 2. Semi-quantitative RT-PCR analysis of RhoGDI2 mRNA. The relative intensity of RhoGDI2 for GAPDH was estimated with computer software (NIH Image 1.63, Wayne Rasband, NIH). The relative expression of RhoGDI2 was compared using the Student's t-test. RhoGDI2 mRNA expression in early gastric cancer was significantly higher compared with normal mucosa and serosa exposed advanced gastric cancer. RhoGDI2, Rho GDP dissociation inhibitor 2; GAPDH, glyceraldehyde 3-phosphate dehydrogenase; RT-PCR, reverse transcription-polymerase chain reaction.

\section{Discussion}

RhoGDIs are known to inhibit the activation of RhoGTPases (15). RhoGDI2 has been established as a metastasis suppressor gene in bladder cancer. Using a gene array analysis, Theodorescu et al reported that decreased RhoGDI2 gene/protein expression was associated with a more invasive variant of the HRAS mutation-positive T24 bladder cancer

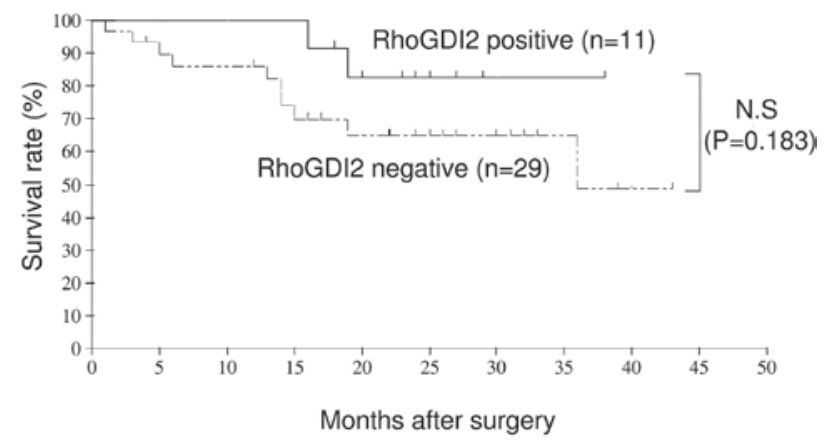

Figure 3. Post-operative relapse-free survival (RFS) of patients with or without RhoGDI2 mRNA expression in gastric carcinoma. RhoGDI2, Rho GDP dissociation inhibitor 2; N.S, not significant.

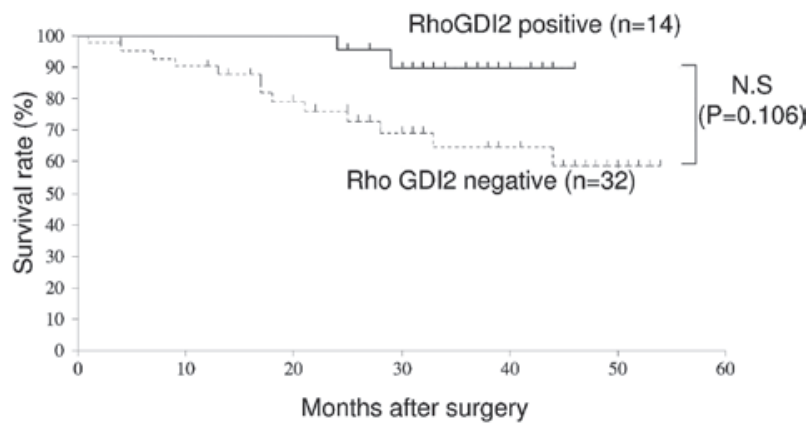

Figure 4. Post-operative overall survival (OS) of patients with or without RhoGDI2 mRNA expression in gastric carcinoma. RhoGDI2, Rho GDP dissociation inhibitor 2; N.S, not significant. 
cell line (12). Ectopic restoration of RhoGDI2 expression in the invasive T24 variant has also been shown to decrease metastasis, as determined by tail-vein lung tumor colonization in mice (10). Finally, the IHC analysis of 51 bladder tumors revealed that RhoGDI2 overexpression correlated with a poor survival time to disease-specific mortality (12). Hu et al identified a biphasic pattern of increased RhoGDI2 expression with breast hyperplasia, but decreased expression with progression and lymph node metastasis using IHC staining in 71 patients (13). In addition, Stevens et al showed that a stable suppression of RhoGDI2 protein expression in ovarian cancer cells increased anchorage-independent growth and Matrigel invasion in vitro, and in tail-vein lung colony metastatic growth in vivo (16). These data show that RhoGDI2 suppresses cancer progression.

Although an increasing number of studies on the role of RhoGDI2 are appearing, the function of RhoGDI2 remains controversial. Tapper et al demonstrated that the upregulation of RhoGDI2 was associated with the malignant potential of ovarian carcinoma by cDNA array analysis (17). An increased motility of murine cancer has been reported to correlate with an overexpression of RhoGDI2 (18). In addition, Cho et al observed that the ectopic overexpression of RhoGDI2 in poorly-invasive gastric carcinoma cell lines significantly increased Matrigel invasiveness in vitro. Conversely, the depletion of endogenous RhoGDI2 in RhoGDI2-overexpressing gastric carcinoma cells suppressed invasion in vitro. A forced expression of RhoGDI 2 in these cells lines increased tumor growth, angiogenesis and lung metastasis in mice (19). The findings indicate that RhoGDI2 is involved in tumorigenesis and cancer progression.

According to the present data, the reduced expression of RhoGDI2 is associated with venous system invasion and lymph node metastasis. The data suggest that RhoGDI2 suppresses cancer metastasis and that the detection of RhoGDI2 mRNA expression may be an useful clinical modality for detecting lymph node metastasis in gastric carcinoma. Dransart et al (20) and Stevens et al (16) found that RhoGDI2 preferentially bound and activated Rac1, and that Rac1 activation antagonized metastasis. Since RhoGDIs are able to modulate Rho GTPase interaction with Rho guanine nucleotide exchange factor (RhoGEFs) and Rho GTPaseactivating proteins (RhoGAPs), RhoGDI2 may activate Rac through the altered regulation of GDP/GTP cycling and then Rac activation may be able to antagonize tumor invasion and metastasis (15).

In summary, the RhoGDI 2 mRNA expression that is usually decreased in advanced-stage gastric cancer was significantly increased in the early-stage gastric cancer patients of the present study. Such expression was almost non-existent in the normal epithelial samples. A reduced expression of RhoGDI2 is associated with venous system invasion and lymph node metastasis, which may lead to the formation of clinical applications for the evaluation of lymph node metastasis in patients with gastric carcinoma.

\section{References}

1. Sahai E and Marshall CJ: RHO-GTPases and cancer. Nat Rev Cancer 2: 133-142, 2002.

2. Clark EA, Golub TR, Lander ES and Hynes RO: Genomic analysis of metastasis reveals an essential role for RhoC. Nature 406: 532-535, 2000.

3. Ueda T, Kikuchi A, Ohga N, Yamamoto J and Takai Y: Purification and characterization from bovine brain cytosol of a novel regulatory protein inhibiting the dissociation of GDP from and the subsequent binding of GTP to rhoB p20, a ras p21-like GTP-binding protein. J Biol Chem 265: 9373-9380, 1990.

4. Leonard D, Hart MJ, Platko JV, et al: The identification and characterization of a GDP-dissociation inhibitor (GDI) for the CDC42Hs protein. J Biol Chem 267: 22860-22868, 1992.

5. Abo A, Pick E, Hall A, Totty N, Teahan CG and Segal AW: Activation of the NADPH oxidase involves the small GTP-binding protein p21rac1. Nature 353: 668-670, 1991.

6. Scherle P, Behrens T and Staudt LM: Ly-GDI, a GDP-dissociation inhibitor of the RhoA GTP-binding protein, is expressed preferentially in lymphocytes. Proc Natl Acad Sci USA 90: 7568-7572, 1993.

7. Lelias JM, Adra CN, Wulf GM, et al: cDNA cloning of a human mRNA preferentially expressed in hematopoietic cells and with homology to a GDP-dissociation inhibitor for the rho GTP-binding proteins. Proc Natl Acad Sci USA 90: 1479-1483, 1993.

8. Leffers H, Nielsen MS, Andersen AH, et al: Identification of two human Rho GDP dissociation inhibitor proteins whose over expression leads to disruption of the actin cytoskeleton. Exp Cell Res 209: 165-174, 1993.

9. Seraj MJ, Harding MA, Gildea JJ, Welch DR and Theodorescu D: The relationship of BRMS1 and RhoGDI2 gene expression to metastatic potential in lineage related human bladder cancer cell lines. Clin Exp Metastasis 18: 519-525, 2000.

10. Gildea JJ, Seraj MJ, Oxford G, et al: RhoGDI2 is an invasion and metastasis suppressor gene in human cancer. Cancer Res 62: 6418-6423, 2002.

11. Gildea JJ, Golden WL, Harding MA and Theodorescu D: Genetic and phenotypic changes associated with the acquisition of tumorigenicity in human bladder cancer. Genes Chromosomes Cancer 27: 252-263, 2000.

12. Theodorescu D, Sapinoso LM, Conaway MR, et al: Reduced expression of metastasis suppressor RhoGDI2 is associated with decreased survival for patients with bladder cancer. Clin Cancer Res 10: 3800-3806, 2004.

13. Hu LD, Zou HF, Zhan SX and Cao KM: Biphasic expression of RhoGDI2 in the progression of breast cancer and its negative relation with lymph node metastasis. Oncol Rep 17: 1383-1389, 2007.

14. Japanese Gastric Cancer Association: Japanese Classification of Gastric Carcinoma - 2nd English Edition. Gastric Cancer 1: 10-24, 1998.

15. Olofsson B: Rho guanine dissociation inhibitors: pivotal molecules in cellular signaling. Cell Signal 11: 545-554, 1999.

16. Stevens EV, Banet N, Onesto C, et al: RhoGDI2 antagonizes ovarian carcinoma growth, invasion and metastasis. Small GTPases 2: 202-210, 2011.

17. Tapper J, Kettunen E, El-Rifai W, et al: Changes in gene expression during progression of ovarian carcinoma. Cancer Genet Cytogenet 128: 1-6, 2001.

18. Yanagawa T, Watanabe H, Takeuchi T, et al: Overexpression of autocrine motility factor in metastatic tumor cells: possible association with augmented expression of KIF3A and GDI-beta. Lab Invest 84: 513-522, 2004.

19. Cho HJ, Baek KE, Park SM, et al: RhoGDI2 expression is associated with tumor growth and malignant progression of gastric cancer. Clin Cancer Res 15: 2612-2619, 2009.

20. Dransart E, Olofsson B and Cherfils J: RhoGDIs revisited: novel roles in Rho regulation. Traffic 6: 957-966, 2005. 